\title{
A MAXIMUM PROPERTY FOR THE CHARACTERISTIC INITIAL VALUE PROBLEM OF THE WAVE EQUATION
}

\section{H. RHEE}

ABSTRACT。A maximum property is obtained for the solutions of the odd-dimensional wave equation in the interior of the characteristic cone.

In this note we will establish a maximum principle for the odd-dimensional wave equation

$$
\square u=\sum_{i=1}^{n} \frac{\partial^{2} u}{\partial x_{i}^{2}}-\frac{\partial^{2} u}{\partial t^{2}}=0, \quad n \text { odd } \geq 3,
$$

in the interior of the characteristic cone $t=|X|, X=\left(x_{1}, x_{2}, \cdots, x_{n}\right)$, under hypotheses on the behavior of $u$ and its derivatives on the cone. A maximum principle for the one-dimensional wave equation $u_{x x}-u_{t t}=0$ in the cone is given in [2].

The ellipsoidal means EM of a continuous function $f(X)$ is defined as follows [1], [3]:

$$
\operatorname{EM}[f ; X, t]=(-1)^{-k}\left(\omega_{n-1} r\right)^{-1}\left(t^{2}-r^{2}\right)^{-k} \int_{b}^{c} d \rho \int_{\beta} \rho^{n-3}\left(1-p^{2}\right)^{k} f(\rho \gamma) d \omega_{\beta},
$$

where $X=r \alpha, r=|X|, t>r, c=(t+r) / 2, b=(t-r) / 2, k=(n-3) / 2, p=t / r+$ $\left(r^{2}-t^{2}\right) / 2 \rho r, \gamma=p \alpha+\left(1-p^{2}\right)^{1 / 2} \beta, \beta$ a unit vector perpendicular to $\alpha, \omega_{n-1}$ $=2 \pi^{(n-1) / 2} / \Gamma((n-1) / 2)$. It is easily seen that

$$
\left(1-p^{2}\right)=\left(r^{2}-t^{2}\right)\left(4 \rho^{2} r^{2}\right)^{-1}(2 \rho+r-t)(2 \rho-r-t) .
$$

Let $\phi(X)$ be a smooth function in $R^{n}$, and let

$$
T \phi(x)=(-1)^{k}(k !)-1 \delta^{k+1}\left(r^{n-3} \phi(r \alpha)\right), \quad \delta=(\partial / \partial r) r .
$$

The method of M. Riesz shows that $u(X, t)=\operatorname{EM}[T \phi ; X, r]$ is the solution of the problem $\square u=0$ in $t>|X|$ with $u(X,|X|)=\phi(X)$.

Using (1), it can be shown that

Received by the editors October 31, 1973 and, in revised form, December 5, 1973. AMS (MOS) subject classifications (1970). Primary 35L05.

Key words and phrases. Wave equation, ellipsoidal means, maximum property, characteristic cone. 


$$
\operatorname{EM}\left[(-1)^{k} ; X, t\right]=\Gamma^{2}(k+1) / \Gamma(2 k+2) .
$$

Hence we have the following maximum principle.

Theorem. Suppose that $\square u=0$ in $t>|X|$ and that $u(X, r) \leq M$. If $(k !)^{-1} \delta^{k+1}\left(r^{n-3} u(X, r)\right)-u(X, r) \leq 0$, then $u(X, t) \leq M$ for $n=3, u(X, t)<M$ for $n \geq 5$ in $t>|X|$.

Remark. It is possible to obtain an explicit representation for the solutions of the even-dimensional wave equation in the cone in terms of given function on the cone. However, the even-dimensional wave equation does not seem to have the above maximum property.

The author would like to thank Professor Hans F. Weinberger for his helpful comments.

\section{REFERENCES}

1. R. Courant and D. Hilbert, Methods of mathematical physics。 II: Partial differential equations, Interscience, New York, 1962. MR 25 \#4216.

2. M. Protter and H. Weinberger, Maximum principles in differential equations, Prentice-Hall, Englewood Cliffs, N. J., 1967. MR 36 \#2935.

3. M. Riesz, L'intégrale de Riemann-Liouville et le problème de Cauchy, Acta Math. 81 (1949), 1-223. MR 10, 713.

DEPARTMENT OF MATHEMATICS, STATE UNIVERSITY OF NEW YORK, COLLEGE AT ONEONTA, ONEONTA, NEW YORK 13820 